\title{
The Effects of the Probiotic Cocktail On Modulation of the NF-kB and JAK/STAT Signaling Pathways Involving in Inflammatory Response in Bowel Disease Model
}

\section{Shadi Aghamohammad}

Pasteur Institute of Iran

\section{Amin Sepehr}

Pasteur Institute of Iran

Seyedeh Tina Miri

Islamic Azad University, Science and Research Branch

\section{Saeideh Najafi}

Islamic Azad University, Science and Research Branch

Mahdi Rohani ( $\square$ kia.rohani@gmail.com )

Pasteur Institute of Iran

Mohammad Reza Pourshafie

Pasteur Institute of Iran

\section{Research Article}

Keywords: Lactobacillus, Bifidobacterium, JAK/STAT, NF-kB, anti-inflammatory

Posted Date: November 1st, 2021

DOI: https://doi.org/10.21203/rs.3.rs-1010470/v1

License: (c) (i) This work is licensed under a Creative Commons Attribution 4.0 International License.

Read Full License 


\section{Abstract}

Background: Probiotics are beneficial microorganisms which have beneficial effects on inflammatory responses, in part, through Janus kinase/signal transduction and activator of transcription (JAK/STAT) and inflammatory signaling pathways.

Materials: Quantitative real-time polymerase chain reaction (qPCR) was used to analyze the expression of JAK/STAT and inflammatory genes (TIRAP, IRAK4, NEMO, and RIP) following treatment of HT-29 cell line with sonicated pathogen, Lactobacillus spp., Bifidobacterium spp. and a mixture cocktail. Cytokine assay also used to evaluate the production of IL- 6 and IL-1B following the probiotic treatment.

Results: Compared to sonicate pathogen treatment cells, our probiotics cocktail was down-regulated JAK genes and TIRAP, IRAK4, NEMO, and RIP genes in NF-kB pathway. The expression of STAT genes were variable following the probiotic treatment. The production of IL- 6 and IL-1B were decreased after probiotic treatment.

Conclusions: Our probiotics cocktail was shown to have anti-inflammatory effects on HT-29 cells by modulating JAK/STAT and NF-kB pathways; therefore, the use of Lactobacillus spp. and Bifidobacterium $s p p$. probiotics as nutritional supplements may reduce inflammation-associated diseases such as inflammatory bowel disease.

\section{Introduction}

The gastrointestinal tract (GT) is an ecosystem containing critical bacteria, including beneficial bacterial genera, which have different effects on the immune system, host metabolism, and improving the microbial balance [1]. According to the World Health Organization (WHO) and the Food and Agriculture Organization (FAO) probiotics are beneficial microorganisms and have perceptible advantageous and limited side effects when used in appropriate amounts and composition [2]. Different genera including Bifidobacteria, Lactobacilli, and other lactic acid bacteria (LAB), such as Lactococci and Streptococci have been extensively studied. In vivo studies revealed that the mixture of Lactobacillus and Bifidobacterium has significant effects on reduction of dysbiosis [3] through regulation of the genes involved in the inflammation [4].

TLR4 and NOD2 are two of the signaling pathway components which recognize the muramyl dipeptide (MDP) and lipopolysaccharide (LPS) of the bacteria. Inflammatory cascades are triggered via activation of genes such as TIR domain-containing adaptor protein (TIRAP), IL-1 receptor-associated kinase (IRAK), and NF-kappa-B essential modulator (NEMO) $[5,6]$. On the other hand, Janus kinase/signal transduction and activator of transcription (JAK/STAT) signaling pathway is one of the major component of both innate and adaptive immune system which mediate cytokines having critical role in the inflammatory diseases [7]. Different components of the JAK/STAT system act through association with various cytokines. STAT1, for instance, is associated with IFN and IL12 whereas STAT3 is associated with IL-6 and IL-10 pathways [8]. In more details, all the components of JAK/STAT system have various roles 
including the enhancement of immunity against infections, differentiation and growths of immune cells, anti-inflammatory actions, and in general have a critical role in haematopoiesis and immunity [9].

Balancing and modulating the immune system and strain-specific anti-inflammatory capacities are two of the critical features of probiotics $[10,11]$. Investigation the effectiveness of Lactobacillus spp. and Bifidobacterium spp. and a mixture of these probiotics on modulating the JAK/STAT and regulation of inflammatory signaling pathways is examined in the present study. Since the greater efficacy could be achieved when different probiotic strains are involved in a mixture, four Lactobacillus spp. and three Bifidobacterium spp. were used in the current study as previously shown to be a definite combination.

\section{Results}

The effectiveness of probiotics in up or down regulation of the inflammatory genes was examined by comparison between treated HT-29 cells with probiotics versus control cells (not exposed HT-29 cells as negative control) and HT-29 cells exposed to sonicated pathogen as positive control. Also, the statistical relatedness between all treatments were examined (figure 2-4). The result of probiotic treatments in JAK/STAT and NF-kB pathway were summarized in figure 1.

\section{The results of stat genes expression}

The data of stat genes expression were showed in figure 1. Comparative analysis of stat1 gene expression between sonicated pathogens and negative control showed that SP-ETEC and SP-ST could increase the gene expression significantly after 48 hours $(p<0.0001)$. Probiotic treatments $(6$ hours before SP tratment) in the first 24 hours decrease significantly the expression level of stat1 gene, while probiotics significantly up-regulated stat 1 gene expression 48 hours after the treatments. There was no significant difference between Lactobacillus spp. alone, Bifidobacterium spp. alone, and Lac/Bif in decreasing or increasing the mRNA level of gene expression.

Using sonictaed pathogens showed the significant increase level in stat2 expression level after 48 hours $(\mathrm{p}<0.01)$. Lactobacillus spp. and Bifidobacterium spp. could significantly decrease the expression level $(\mathrm{p}<0.001)$, in comparison with positive control (SP48) after 24 hours of treatment, while Lactobacillus spp. (LP48) had the most significant effects on increasing the expression level $(p<0.0001)$.

Comparative analysis of stat3 gene expression between sonicated pathogens and negative control showed that SP-ETEC and SP-ST could increase the gene expression, significantly $(p<0.0001)$. Using probiotic treatments ( 6 hours before SP treatment) showed that all the versions could increase the gene expression level, except for the treatment of Lactobacillus spp. after 24 hours (LP24). The most effective treatment in increasing the level of gene expression was Lactobacillus spp. after 48 hours of treatment $(L P 48)(p<0.0001)$. In all formats of probiotic-SP treatments, increasing the gene expression level was more significant after 48 hours of treatments $(p<0.0001$ and $p<0.001)$. 
Treatments with sonicated pathogens showed the increased level of stat4 expression level; although the results of probiotic treatments were variable. Using Lactobacillus spp-SP. ,either after 24 or 48 hours, could increase the gene expression, significantly $(p<0.0001)$. Vice versa, Bifidobacterium sppSP. decreased the stat 4 expression level both after 24 and 48 hours of treatments $(p<0.0001)$. Using Lac/Bif-SP could decrease the expression level in the first 24 hours of treatment $(p<0.0001)$.

Comparative analysis of stat 5 gene expression between sonicated pathogens and negative control showed that SP-ETEC and SP-ST could increase the gene expression, significantly, specifically after 24 hours. All the probiotic treatments ( 6 hours before SP treatment) decreased stat 5 expression level $(p<$ 0.0001), except for Lactobacillus spp. after 48 hours of treatment (LP48), that increase the mRNA level ( $p$ $<0.0001)$. Lactobacillus spp. had more significant effects on decreasing the gene expression in the first 24 hours of treatment (LP24).

After using sonicated pathogene, the expression level of stat6 increased, significantly after 48 hours of treatments $(p<0.0001)$. All the versions of probiotic treatmnets ( 6 hours before SP treatment) decreased the expression level, in comparison with positive control (SP48). Bifidobacterium spp. and Lac/Bif in the first 24 hours of treatment had the most significant effects on decreasing the expression level Fig 1.

\section{The results of jak genes expression}

The data of jak esxpressions was showed in figure 2. The results of jak expression level showed more homogenous outcomes. The results of both jak1 and jak3 showed that the treatment of sonicated pathogen could increase the expression level after 24 hours of treatment $(p<0.0001)$. All the versions of probiotic treatment decrease the mRNA level of gene expression. There was no significant difference between probiotic treatment.

Comparative analysis of jak2 gene expression indicated that sonicated pathogens increased the gene expression. All the probiotic treatments (6 hours before SP treatment) decrease the mRNA level of gene expression. Lac/Bif treatment had the lowest effect on decreasing the gene expression.

After using SP in the first 24 hours of treatment, the expression level of tyk2 was increased $(p<0.0001)$. All the probiotic treatments ( 6 hours before SP treatment), except for Lactobacillus spp. after 48 hours of treatment (LP48), could decrease the gene expression and Bifidobacterium spp. had the most significant effect Fig 2.

\section{The results of inflammatory genes expression}

The data of inflammatory gene esxpressions was showed in figure 3 . The analysis of inflammatory genes including tirap, irak4, rip, and nemo resulted in up-regulation following treatment of SP-ETEC and SP-ST. In contrast, probiotics treatments down-regulated the inflammatory genes. Lactobacillus spp. and Bifidobacterium spp. had more significant effects on decreasing nemo expression level in comparison with Lac/Bif treatments. For other inflammatory genes, there was no significant difference between probiotic treatments Fig 3. 


\section{The result of pro-inflammatory cytokines production}

The production of cytokines was significantly higher after SP treatments. however, probiotic treatments (6 hours before SP treatment) significantly could decreased cytokine production. No significant difference between Lactobacillus spp, Bifidobacterium spp, and Lac/Bif was seen at any time after treatment Fig 4.

\section{Discussion}

The use of probiotics in immunologically related diseases such as IBD has been studied based on an idea that probiotics can modulate the immune system [12]. Imbalance of pro-inflammatory and antiinflammatory signaling pathways have been the main observation in IBD [13]. It has been shown that colitis and ulcerative colitis diseases, two types of IBD, are associated with different types of cytokines. Colitis can activate Th1 cells resulting in release of Th1- related cytokines such as IFN- $\gamma$, while ulcerative colitis is associated with Th2 and release of IL-5, IL-13, and IL-9. Therefore, finding the possible way to reduce the signaling molecules resulted in producing these cytokines would be a potential treatment in managing of IBD by decrease the severity of symptoms [14]. Probiotics are useful bacteria to have major roles in prevention and treatment of IBD [15]. The probiotic strains which have been used in this study, previously shown in our laboratory to have phenotypic anti-inflammatory effects [1]. To understand the genetics of such anti-inflammatory responses, we examined the NF-kB and JAK/STAT signaling pathways following probiotic treatment of HT-29 cell line. On the other hand, we decided to use probiotics as pre-treatments ( 6 hours before SP-treatment) to evaluate the role of probiotics as preventive agents.

Analysis of NF-kB signaling pathway showed the significant decrease of inflammatory genes with probiotic treatments. Comparing of HT-29 cells exposed with SP with our probiotic strains showed reverse effects. On one hand, SP resulted in increase in expression of the inflammatory genes involved in NF-kB signaling pathway, whereas our probiotics strains in all forms, alone or mixture, resulted in decrease of the mRNA level of the studied inflammatory genes. This may explain the molecular mechanism of our finding that was reported in previous study [1], where we showed significant reduction in IBD-induced mice inflammatory responses following exposure with these selected probiotics. In support of this notion, it was found that the expression level of rip gene following treatment with SP, resulted in increasement of expression. rip, as one of the components of the NOD2 signaling pathway, has a critical role in the production of cytokines and causing inflammation in IBD as well [16]. However, when probiotics were added to HT-29 cells, 6 hours before inducing the inflammation, more than 7 folds decreases in expression of rip gene was observed, suggesting the effectiveness of our probiotic spp. in controlling rip gene which, in turn, could affect the production of inflammatory cytokines.

tirap (another studied inflammatory gene) is one of the key components of NF-kB signaling pathway and different studies reported that mutation of tirap could result in a reduction of cytokine production [17]. Here, tirap in HT-29 cells was decreased more significantly than the other three genes, including nemo, irak4, and rip. Almost no expression of tirap gene was seen following treatment of HT-29 cell line with Lactobacillus spp. or Bifidobacterium spp. or Lac/Bif after 48 hours of treatment, suggesting that our 
probiotics strains could interfere with cytokine production specifically if the duration usage of probiotics would be longer. Moreover, our preliminary examination of the supernatants of HT-29 cells treated with probiotics showed significant reduction of IL- 6 and IL-1 $\beta$.

Besides NF-kB pathway, JAK/STAT plays various roles associated with different types of cytokines which could affect the status of IBD [18]; however, The roles of STATs in inflammatory diseases, including IBD, are complicated. Taken together, STATs as immunological factors help to reduce the severity of IBD and improved the inflammatory status. The STAT1, for instance, could be up-regulated as a defensive reaction so that the inflammation would be limited. STAT3, another component of the STAT group, is also critical in IBD since it could be associated with some anti-inflammatory cytokines, including IL-10 and IL-22. Although STAT3 acts in a pro-inflammatory mode in the adaptive immune system, it becomes appeared as protective agents in the innate immune system. In fact, STAT3 play important role in the cellular stress response, apoptosis, and the processes which involved in wound healing in intestinal epithelial cells. Totally, different components of the STAT family (specifically STAT1, 3, and 5) could affect IBD via activating anti-inflammatory components, promote the maintenance of integrity, and regeneration of crypt epithelium [19]. Generally, the components of JAK/STAT family, including STAT3 and JAK1, could be affected by different ligands like IL-10 and IL-6, as anti and pro-inflammatory cytokines; therefore the JAK/STAT family could play various roles in immune homeostasis [20]. Coskun et al., for example, reported different roles of STAT3 in diverse cell types, noted the complexity and opposite actions of JAK/STAT family [21]. In the current study, we had different result in the expression of STAT genes. Some probiotic treatments up and the others down regulated the gene expression level. In STAT1, 2 and 4 both up and down regulation was seen. STAT3 and STAT 5 were approximately up and STAT6 6 was downregulated. This because, as said above, STATs have various roles in immune homeostasis and probiotic also could have immune modulation effects in different diseases like IBD [22]; therefore all these variation helped to improve the condition of inflammation and are in coordination with other studies. Also, studies by other investigators have shown that increased STAT6 could play a significant role in the colitis by effecting the production of IL-13 [23], whereas decrease of STAT6 could prevent apoptosis and disruption of cell membrane integrity [24]. About JAK expression, our result showed that probiotics downregulated the expression of JAK genes. Here our probiotic exhibited anti-inflammatory function similar to JAK inhibitors such as JAKinibs a drug which target JAK by reducing JAK expression [25]. One point that should be noted is that, although Lactobacillus spp, Bifidobacterium spp, and Lac/Bif had different roles in each gene, Lactobacillus spp. overall had the most effects on gene expressions. In brief, all tests, including molecular examinations and phenotypic study (cytokine assay), showed decreased the inflammatory status. In general, because we use probiotics as pre-treatment, the results may suggest that the use of probiotic could, in turn, results in preventing the severity of IBD.

\section{Material And Methods}

\section{Bacterial strain, Culture medium and Growth conditions}


In this study, 4 Lactobacillus spp. including L. plantarum, L. rhamnosus, L. brevis, and L. reuteri were isolated from the fecal samples of healthy individuals [1] and 3 Bifidobacterium spp. including B. bifidum, $B$. longum and $B$. infantis were isolated from breast milk as reported before [26]. The probiotic and phenotypic characteristics of these strains were examined before $[1,26]$. The bacteria were inoculated into MRS broth containing $0.05 \% \mathrm{~L}$-cysteine and incubated for $20 \mathrm{~h}$ at $37^{\circ} \mathrm{C}$. Furthermore, pathogenic bacteria, including enterotoxigenic Escherichia coli (ETEC) and Salmonella typhimurium (ST) were cultured on Luria-Bertani (LB) broth (Thermo Fisher Scientific, US) followed by sonication to disrupt the cells' integrity. In order to obtain crude sonicated bacterial preparation, heat-killed bacteria were sonicated (10 rounds, $1 \mathrm{~min} /$ round) and the cellular debris was centrifuged $\left(1700 \mathrm{~g}, 15 \mathrm{~min}, 4^{\circ} \mathrm{C}\right)$ resulting in sonicated pathogen (SP). All methods were carried out in accordance with relevant guidelines and regulations, the fecal samples and breast milk have been obtained from the previous study and ethical approval for the previous study is obtained from the committee of Pasteur Institute of Iran (IR.PII.REC.1398.060). Sign informed consent was obtained from all participants.

\section{Treatment of HT-29 cells with probiotics}

Human colon adenocarcinoma cell line HT-29 was obtained from the Cell Bank of Pasteur Institute of Iran. HT-29 cells were grown in RPMI-1640 (Thermo-Gibco, USA) supplemented with $10 \%$ fetal bovine serum (Biochrom, Berlin, Germany), and 1\% penicillin-streptomycin (Sigma_Aldrich, UK). To perform the different treatments, cells were detached by $0.25 \%$ Trypsin-EDTA (Gibco, USA), washed twice with PBS and counted. Cell suspension was centrifuged and the precipitate was diluted with RPMI-1640 and 2x10 cells per well were seeded. For preparing the bacterial suspension, the culture pellet was collected and diluted in RPMI-1640 with 10\% FBS without antibiotic to reach the final optical density concentration (OD) of 0.08 at $600 \mathrm{~nm}$. For Lactobacillus/Bifidobacterium mixture (Lac/Bif), equal amounts of the prepared solutions adjusted with OD of 0.08 at $600 \mathrm{~nm}$ of each bacterium after which they were mixed.

HT-29 cells were exposed to different bacteria, either alone or in combinations, including sonicated pathogen enterotoxigenic E. coli (SP-ETEC), sonicated pathogen Salmonella typhi(SP-ST), Lactobacillus spp. alone, Bifidobacterium spp. alone, Lactobacillus/Bifidobacterium mixture (Lac/Bif). In order to examine the effects of the probiotic treatments on HT-29 cells upon exposure with SP, different combinational treatments were done. The treatments of HT-29 cells were done as follow: Lactobacillus spp., Bifidobacterium spp. and the Lac/Bif were added to the HT-29 cell line and after 1 hour each wells were washed twice with PBS for excluding the non-attached bacteria and then SP-ETEC and SP-ST were added. These treatments were done in duplicates and examined at 24- and 48-hours post-exposure. The 6 well culture plate was incubated at $37^{\circ} \mathrm{C}$ and $5 \% \mathrm{CO}_{2}$. After 24 and $48 \mathrm{hrs}$, the cells were harvested by $0.25 \%$ Trypsin-EDTA, washed twice with PBS, centrifuged and RNA was extracted. Determination of the multiplicity of infection (MOI) was done as indicated previously [27].

\section{RT- PCR of inflammatory signaling pathway genes}


Total RNA was extracted using a total RNA extraction kit (Roche, Germany) according to the manufacturer's instructions. The quantity and quality of the purified RNA were determined by a NanoDrop1000 UV-Vis Spectrophotometer (measuring the absorbance at 260/280 nm). The cDNA template was synthesized with the cDNA synthesis kit (Yekta Tajhiz, Iran) according to the manufacturer's instructions. The online Primer-Bank website (http://pga.mgh.harvard.edu/primerbank) was used to choose the qPCR primers (Table 1). All the primers were tested using gradient PCR to get appropriate annealing temperature. The mRNA quantification of studied genes was evaluated with ABI step one plus detection system (Applied Bio systems, USA co) using SYBR Green master mix (Amplicon Bio, Denmark). All the reactions were performed in duplicate. The formula $R Q=2^{-\Delta \Delta C t}$ was used to get relative gene expression is the comparative CT method [28]. The appropriate internal control gene, glyceraldehyde 3phosphate dehydrogenase ( $g a p d h)$, was selected as a housekeeping gene to normalize the data.

\section{Cytokine assays}

After performing treatments, the supernatant of the cell culture was centrifuged at $6000 \mathrm{rpm}$, sediment was discarded and the supernatant was collected to evaluate the production of pro-inflammatory cytokines, including IL- 6 and IL-1B by Elisa kit (Karmanian Pars Gene, Iran) according to the manufacturer's protocols.

\section{Statistical analysis}

Graphs and statistical analysis of the data were performed using SPSS (ver.25) and REST 2009 software to compare variables of different groups. Statistical differences in multiple groups were determined using ordinary one-way ANOVA. $P$-values $<0.05$ were considered as statistically significant. The results were presented as mean \pm Standard Error of Mean (SEM).

\section{Declarations}

\section{Ethics approval and consent to participate}

The experimental protocols were established following the Declaration of Helsinki and approved by the ethics committee of Pasteur Institute of Iran (IR.PII.REC.1398.060). Signed informed consent was obtained from all participants.

\section{Consent for publication}

Not applicable.

\section{Data Availability Statement}

The datasets generated during and/or analyzed during the current study are available from the corresponding author on reasonable request. 


\section{Competing interests}

The authors declare no conflict of interest.

\section{Funding}

The current research was done in support of Pasteur Institute of Iran supported as funding agency (with grant number 1694).

\section{Authors contributions}

Performed the experiments: ShA, SM, SN, Data analysis: AS, SM, ShA, Writing of the manuscript: ShA, AS, Revised manuscript MP, MR, and Conceived and designed the experiments: MP, MR

\section{Acknowledgments}

The authors would like to thank Pasteur Institute of Iran as funding agency (with grant number 1694) and Dr. Sana Eybpoosh in the Department of Epidemiology and Biostatistics Research Centre for Emerging and Reemerging Infectious Diseases, Pasteur Institute of Iran, Tehran, for her help and support.

\section{References}

1. Rohani M, Noohi N, Talebi M, Katouli M, Pourshafie MR: Highly heterogeneous probiotic Lactobacillus species in healthy Iranians with low functional activities. PloS one 2015, 10(12):e0144467.

2. Hoseinifar SH, Sun Y-Z, Wang A, Zhou Z: Probiotics as means of diseases control in aquaculture, a review of current knowledge and future perspectives. Frontiers in Microbiology 2018, 9:2429.

3. Azad M, Kalam A, Sarker M, Li T, Yin J: Probiotic species in the modulation of gut microbiota: an overview. BioMed research international 2018, 2018.

4. Salas A, Hernandez-Rocha C, Duijvestein M, Faubion W, McGovern D, Vermeire S, Vetrano S, Casteele NV: JAK-STAT pathway targeting for the treatment of inflammatory bowel disease. Nature Reviews Gastroenterology \& Hepatology 2020, 17(6):323-337.

5. Li J, Li B, Cheng Y, Meng Q, Wei L, Li W, Zhang J, Huang S: The synergistic effect of NOD2 and TLR4 on the activation of autophagy in human submandibular gland inflammation. Journal of Oral Pathology \& Medicine 2019, 48(1):87-95.

6. Moen SH, Ehrnström B, Kojen JF, Yurchenko M, Beckwith KS, Afset JE, Damås JK, Hu Z, Yin H, Espevik T: Human Toll-like receptor $\mathbf{8}$ (TLR8) is an important sensor of pyogenic bacteria, and is attenuated by cell surface TLR signaling. Frontiers in immunology 2019, 10:1209.

7. Shi H, Deng X, Deng Q, Liu Z, Liu N: Probiotic Lactobacilli Improved Growth Performance and Attenuated Salmonella Typhimurium Infection Via Jak/Stat Signaling in Broilers. Brazilian Journal of Poultry Science 2021, 23. 
8. Yousefi B, Eslami M, Ghasemian A, Kokhaei P, Salek Farrokhi A, Darabi N: Probiotics importance and their immunomodulatory properties. Journal of cellular physiology 2019, 234(6):8008-8018.

9. Schwartz DM, Bonelli M, Gadina M, O'shea JJ: Type I/II cytokines, JAKs, and new strategies for treating autoimmune diseases. Nature Reviews Rheumatology 2016, 12(1):25-36.

10. Alard J, Peucelle V, Boutillier D, Breton J, Kuylle S, Pot B, Holowacz S, Grangette C: New probiotic strains for inflammatory bowel disease management identified by combining in vitro and in vivo approaches. Beneficial microbes 2018, 9(2):317-331.

11. Zhang H, Yeh C, Jin Z, Ding L, Liu BY, Zhang L, Dannelly HK: Prospective study of probiotic supplementation results in immune stimulation and improvement of upper respiratory infection rate. Synthetic and systems biotechnology 2018, 3(2):113-120.

12. Amer M, Nadeem M, Nazir R, Ur S, Fakhar M, Abid F, Asif E, Nazir SUR, Ain Q-U: Probiotics and their use in inflammatory bowel disease. Alternative Therapies in Health \& Medicine 2018, 24(3).

13. Wang H, Gu J, Hou X, Chen J, Yang N, Liu Y, Wang G, Du M, Qiu H, Luo Y: Anti-inflammatory effect of miltirone on inflammatory bowel disease via TLR4/NF-kB/IQGAP2 signaling pathway. Biomedicine \& Pharmacotherapy 2017, 85:531-540.

14. Marafini I, Sedda S, Dinallo V, Monteleone G: Inflammatory cytokines: from discoveries to therapies in IBD. Expert opinion on biological therapy 2019, 19(11):1207-1217.

15. Jakubczyk D, Leszczyńska K, Górska S: The effectiveness of probiotics in the treatment of inflammatory bowel disease (IBD)-a critical review. Nutrients 2020, 12(7):1973.

16. Speir M, Djajawi TM, Conos SA, Tye H, Lawlor KE: Targeting RIP Kinases in Chronic Inflammatory Disease. Biomolecules 2021, 11(5):646.

17. Fawkner-Corbett D, Simmons A, Parikh K: Microbiome, pattern recognition receptor function in health and inflammation. Best practice \& research Clinical gastroenterology 2017, 31(6):683-691.

18. Gulamhusein AF, Eaton JE, Tabibian JH, Atkinson EJ, Juran BD, Lazaridis KN: Duration of inflammatory bowel disease is associated with increased risk of cholangiocarcinoma in patients with primary sclerosing cholangitis and IBD. The American journal of gastroenterology 2016, 111(5):705.

19. Zundler S, Neurath MF: Integrating immunologic signaling networks: the JAK/STAT pathway in colitis and colitis-associated cancer. Vaccines 2016, 4(1):5.

20. Soendergaard C, Bergenheim FH, Bjerrum JT, Nielsen OH: Targeting JAK-STAT signal transduction in IBD. Pharmacology \& therapeutics 2018, 192:100-111.

21. Coskun M, Salem M, Pedersen J, Nielsen $\mathrm{OH}$ : Involvement of JAK/STAT signaling in the pathogenesis of inflammatory bowel disease. Pharmacological research 2013, 76:1-8.

22. Kang H-J, Im S-H: Probiotics as an immune modulator. Journal of Nutritional Science and Vitaminology 2015, 61(Supplement):S103-S105.

23. Delgado-Ramirez Y, Ocaña-Soriano A, Ledesma-Soto Y, Olguín JE, Hernandez-Ruiz J, Terrazas LI, Leon-Cabrera S: STAT6 Is Critical for the Induction of Regulatory T Cells In Vivo Controlling the Initial 
Steps of Colitis-Associated Cancer. International Journal of Molecular Sciences 2021, 22(8):4049.

24. Rosen MJ, Frey MR, Washington KM, Chaturvedi R, Kuhnhein LA, Matta P, Revetta FL, Wilson KT, Polk BD: STAT6 activation in ulcerative colitis: a new target for prevention of IL-13-induced colon epithelial cell dysfunction. Inflammatory bowel diseases 2011, 17(11):2224-2234.

25. Villarino AV, Kanno Y, O'Shea JJ: Mechanisms and consequences of Jak-STAT signaling in the immune system. Nature immunology 2017, 18(4):374-384.

26. Eshaghi M, Bibalan MH, Rohani M, Esghaei M, Douraghi M, Talebi M, Pourshafie MR: Bifidobacterium obtained from mother's milk and their infant stool; A comparative genotyping and antibacterial analysis. Microbial pathogenesis 2017, 111:94-98.

27. Ghanavati R, Asadollahi P, Shapourabadi MB, Razavi S, Talebi M, Rohani M: Inhibitory effects of Lactobacilli cocktail on HT-29 colon carcinoma cells growth and modulation of the Notch and Wnt/ $\beta$ catenin signaling pathways. Microbial pathogenesis 2020, 139:103829.

28. Schmittgen TD, Livak KJ: Analyzing real-time PCR data by the comparative CT method. Nature Protocols 2008, 3(6):1101-1108.

\section{Tables}

Table 1. Primer sequences used in this study 


\begin{tabular}{|c|c|c|c|}
\hline Gene & Primer Sequence [5' > 3'] & Primer Bank ID & Product Size [bp] \\
\hline STAT1 F & CGGCTGAATTTCGGCACCT & $189458859 c 3$ & 81 \\
\hline STAT1 R & CAGTAACGATGAGAGGACCCT & & \\
\hline STAT2 F & CTGCTAGGCCGATTAACTACCC & $291219923 c 3$ & 87 \\
\hline STAT2 R & TCTGATGCAGGCTTTTTGCTG & & \\
\hline STAT3 F & ACCAGCAGTATAGCCGCTTC & $47080104 c 2$ & 124 \\
\hline STAT3 R & GCCACAATCCGGGCAATCT & & \\
\hline STAT4 F & GCTTAACAGCCTCGATTTCAAGA & $345110659 c 2$ & 91 \\
\hline STAT4 R & GAGCATGGTGTTCATTAACAGGT & & \\
\hline STAT5 F & CGACGGGACCTTCTTGTTG & $221316717 c 3$ & 80 \\
\hline STAT5 R & GTTCCGGGGAGTCAAACTTCC & & \\
\hline STAT6 F & CGAGTAGGGGAGATCCACCTT & $296010867 c 2$ & 92 \\
\hline STAT6 R & GCAGGAGTTTCTATCAAGCTGTG & & \\
\hline JAK1 F & CTTTGCCCTGTATGACGAGAAC & $102469033 c 1$ & 101 \\
\hline JAK1 R & ACCTCATCCGGTAGTGGAGC & & \\
\hline JAK2 F & ATCCACCCAACCATGTCTTCC & $223671934 c 2$ & 121 \\
\hline JAK2 R & ATTCCATGCCGATAGGCTCTG & & \\
\hline JAK3 F & CTGCACGTAGATGGGGTGG & $189095272 c 2$ & 78 \\
\hline JAK3 R & CACGATCAGGTTGGACTTTTCT & & \\
\hline TYK2 F & GAGATGCAAGCCTGATGCTAT & $187608614 c 1$ & 76 \\
\hline TYK2 R & GGTTCCCGAGGATTCATGCC & & \\
\hline RIP2 F & GCCCTTGGTGTAAATTACCTGC & $93141034 c 2$ & 138 \\
\hline RIP2 R & GGACATCATGCGCCACTTT & & \\
\hline NEMO F & AAGAGCCAACTGTGTGAGATG & $142381344 c 1$ & 69 \\
\hline NEMO R & TTCGCCCAGTACGTCCTGA & & \\
\hline TIRAP F & GACCCCTGGTGCAAGTACC & $89111123 c 2$ & 133 \\
\hline TIRAP R & CGACGTAGTACATGAATCGGAG & & \\
\hline IRAK4 F & CTTGGATGGTACTCCACCACT & $223671887 c 3$ & 76 \\
\hline IRAK4 R & AAAATTGATGCCATTAGCTGCAC & & \\
\hline
\end{tabular}




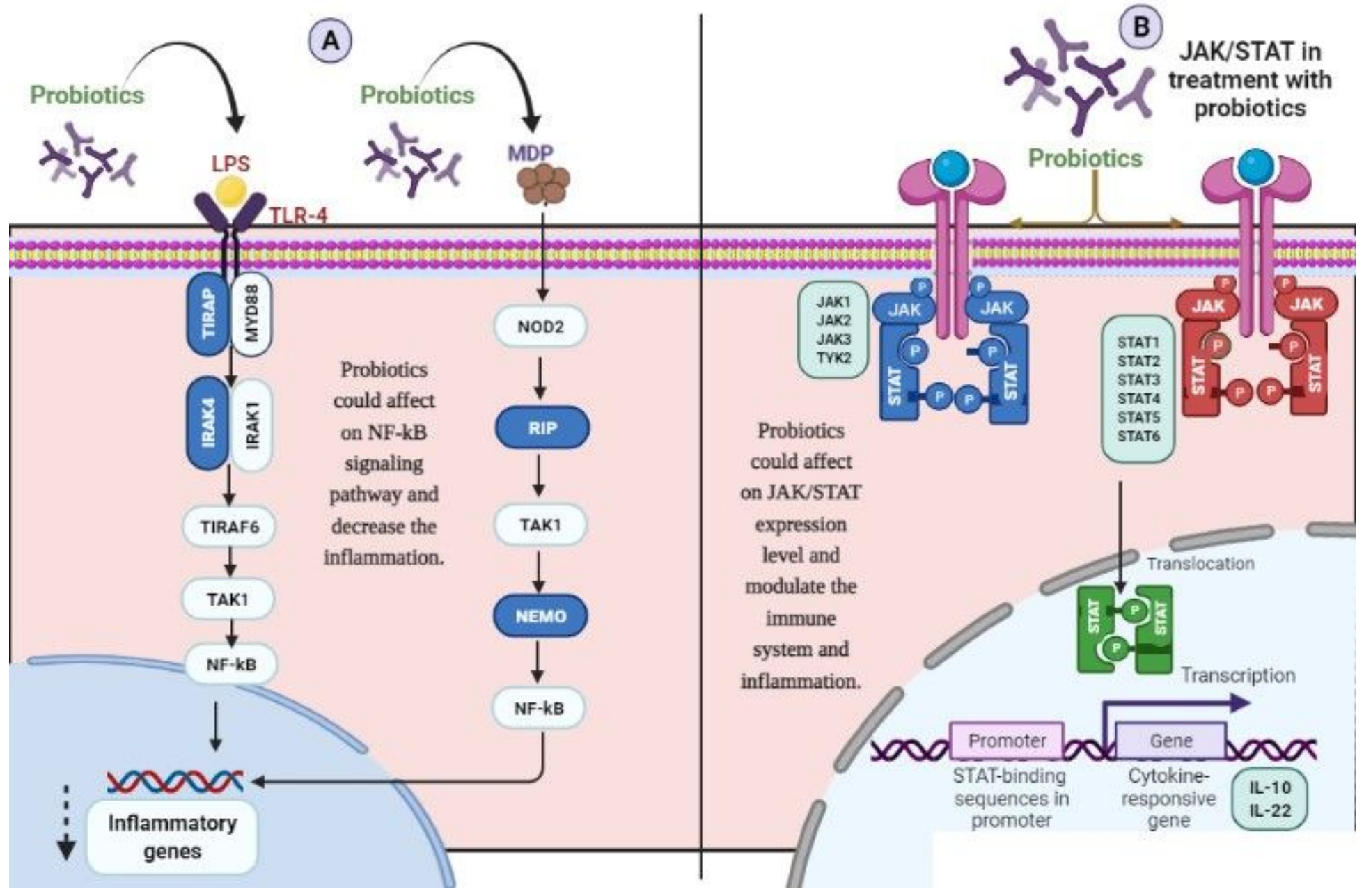

Figure 1

The overal result of probiotic treatments in A) NF-kB pathway and B) JAK/STAT. 

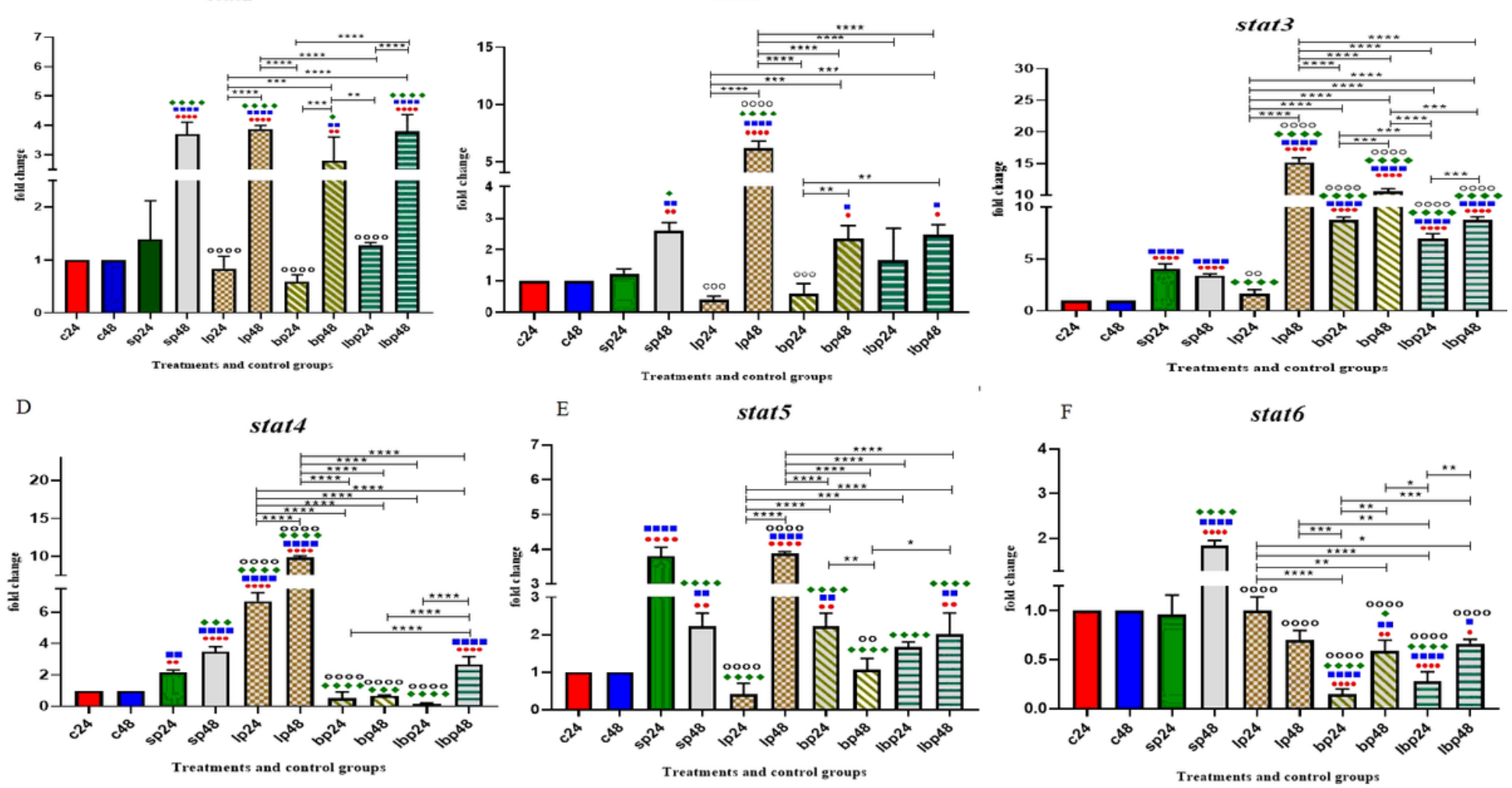

Figure 2

Relative gene expression (mean fold change) of (a) stat1, (b) stat2, (c) stat3, (d) stat4, (e) stat5, and (F) stat6 in the different groups of treatments. Data were normalized with gapdh. Data were represented as mean SD. The number of 24 and 48 refers to different time orders of HT-29 cell line treatments. C, control; P, Pathogen; LP, first Lactobacillus spp. and then pathogen; BP, first Bifidobacterium spp. and then pathogen; LBP, first Lac/Bif and then pathogen. Data were considered as statistically significant when $p<$ $0.05\left({ }^{*} p<0.05,{ }^{*} p<<0.01,{ }^{* \star *} p<0.001\right.$ and $\left.{ }^{* \star *} \mathrm{p} p<0.0001\right)$. Red circle indicates the relatedness between C24 with other treatments, Blue Square shows the relatedness between C48 and other treatments, Green diamond indicates the relatedness between P24 with other treatments, and empty circle shows the relatedness between P48 with other treatments. The relatedness between other treatments are shown with brackets. 
A

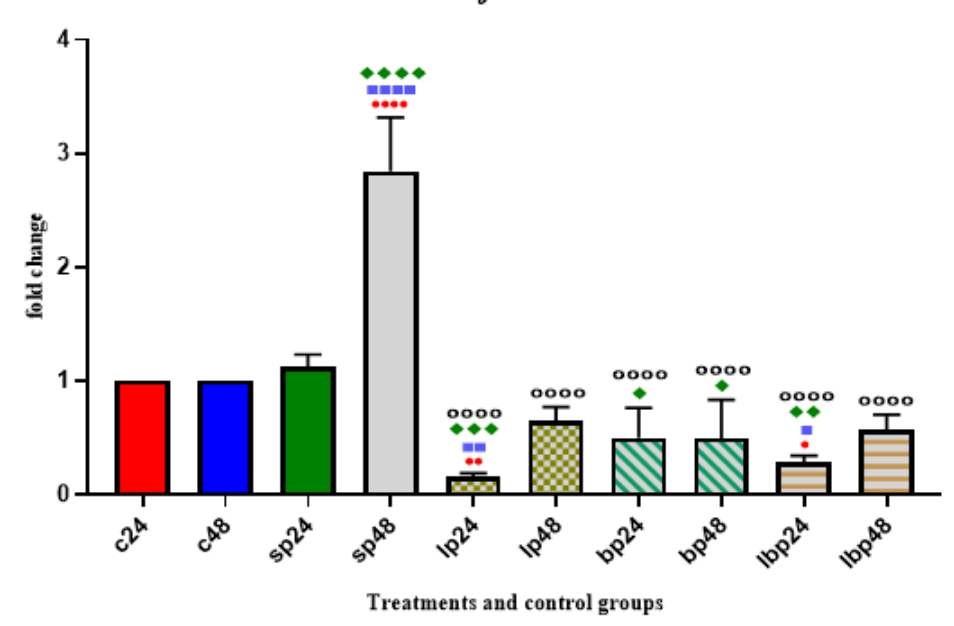

$\mathrm{C}$

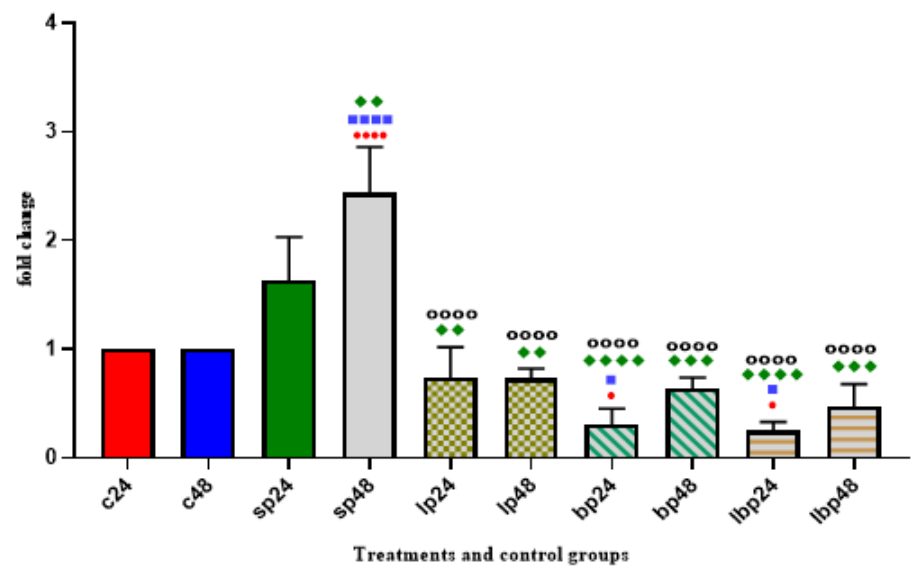

B
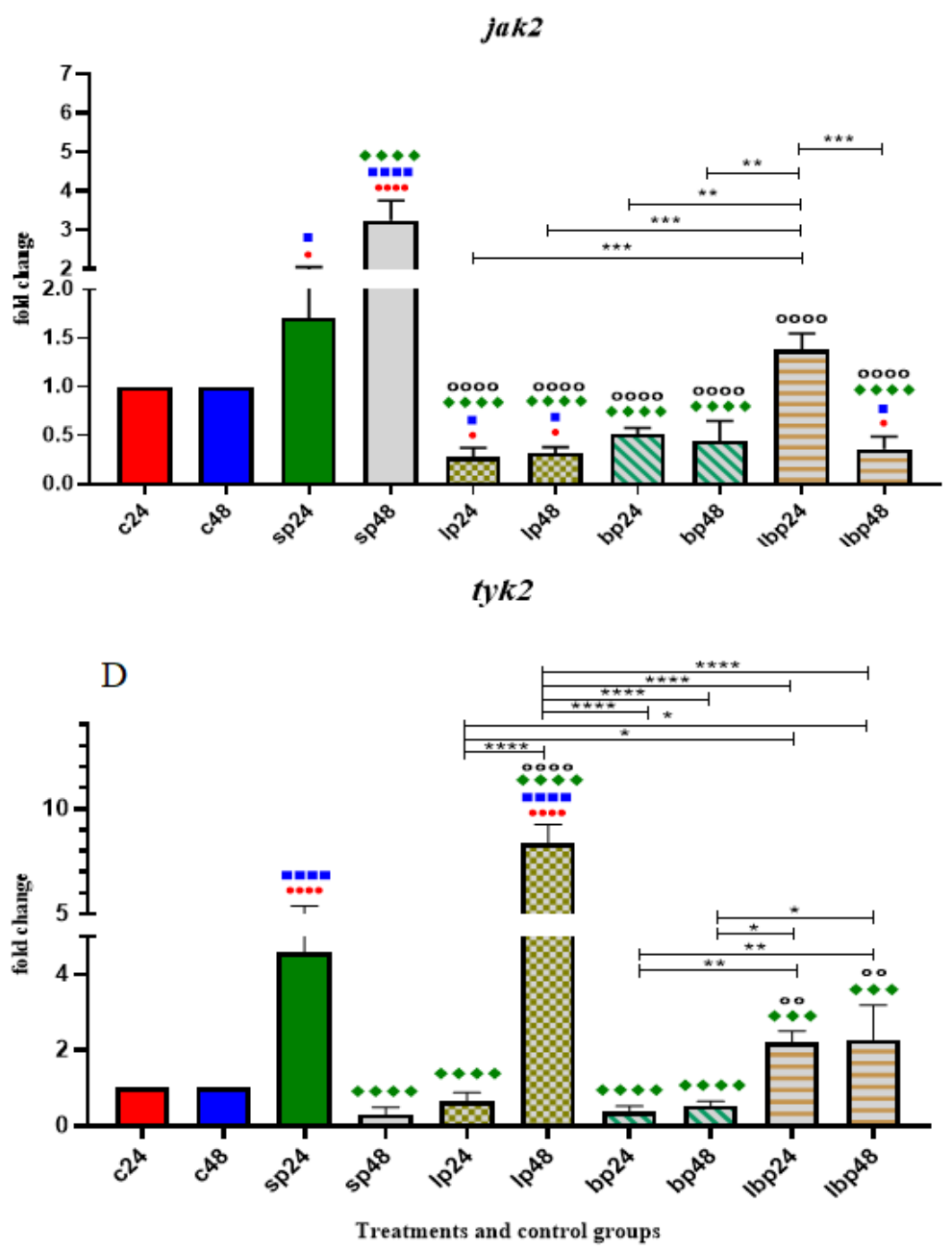

Figure 3

Relative gene expression [mean fold change] of [a] jak1, [b] jak2, [c] jak3, [d], and tyk2 in the different groups of treatments. Data were normalized with gapdh. Data were represented as mean SD. The number of 24 and 48 refers to different time orders of HT-29 cell line treatments. C, control; P, Pathogen; LP, first Lactobacillus spp. and then pathogen; BP, first Bifidobacterium spp. and then pathogen; LBP, first Lac/Bif and then pathogen. Data were considered as statistically significant when $p<0.05\left[{ }^{\star} p<0.05,{ }^{\star \star} p<0.01\right.$, $\star \star \star * p<0.001$ and $\left.{ }^{* \star * \star} p<0.0001\right]$. Red circle indicates the relatedness between $\mathrm{C} 24$ with other treatments, Blue Square shows the relatedness between $\mathrm{C} 48$ and other treatments, Green diamond indicates the relatedness between P24 with other treatments, and empty circle shows the relatedness between P48 with other treatments. The relatedness between other treatments are shown with brackets. 
A

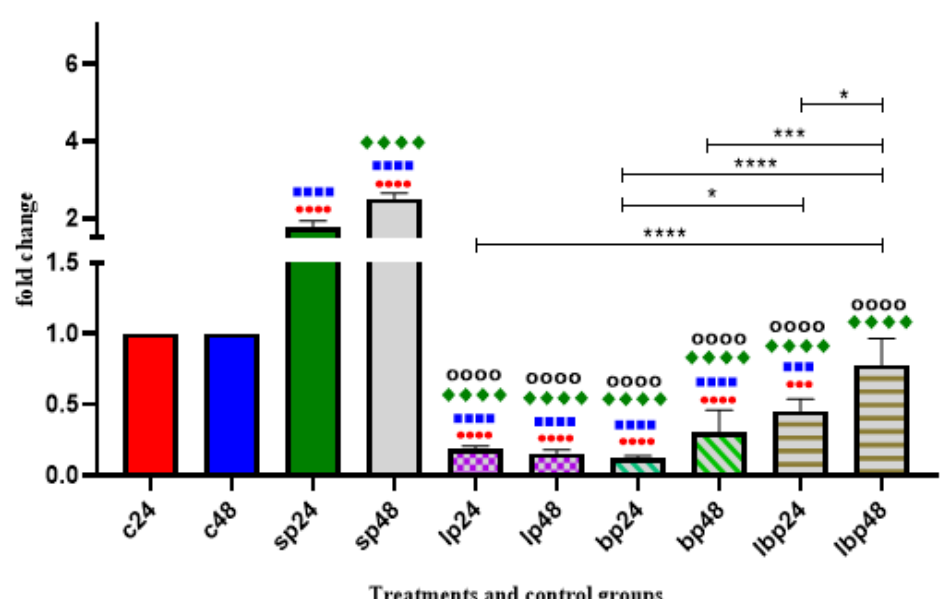

Treatments and control groups

$\mathrm{C}$

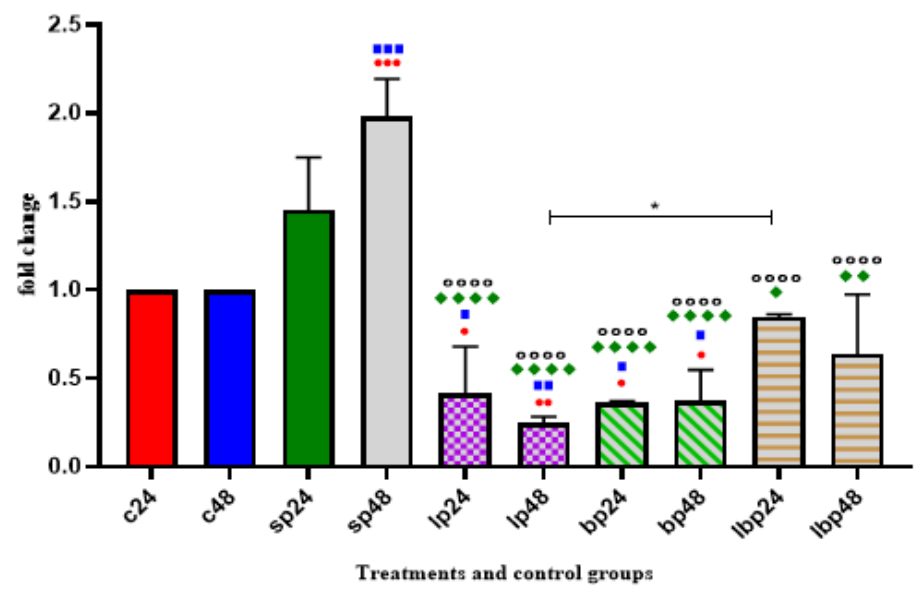

tirap

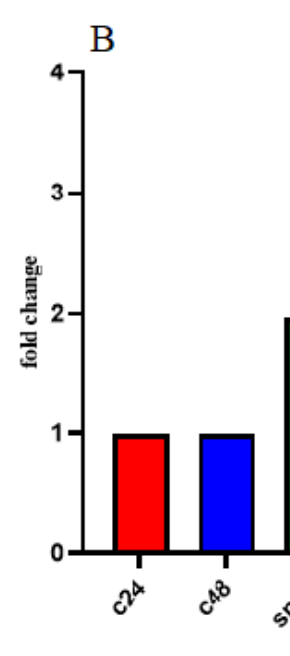

$\mathrm{D}$

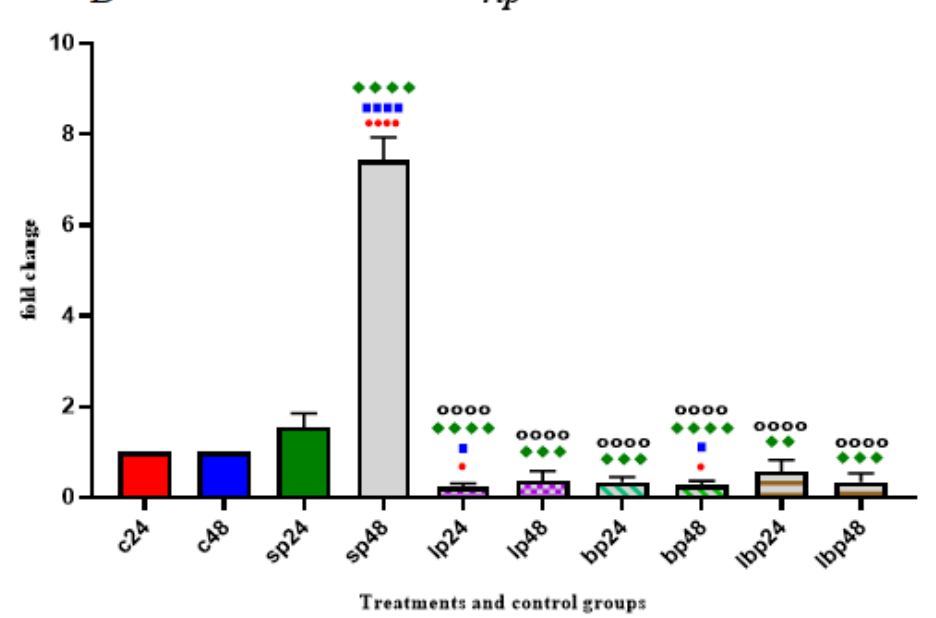

Figure 4

Relative gene expression [mean fold change] of [a] nemo, [b] tirap, [c] irak, [d], and rip in the different groups of treatments. Data were normalized with gapdh. Data were represented as mean SD. The number of 24 and 48 refers to different time orders of HT-29 cell line treatments. C, control; P, Pathogen; LP, first Lactobacillus spp. and then pathogen; BP, first Bifidobacterium spp. and then pathogen; LBP, first Lac/Bif and then pathogen. Data were considered as statistically significant when $p<0.05\left[{ }^{\star} p<0.05\right.$, ${ }^{\star \star} p<0.01$, ${ }^{\star * \star} \mathrm{p}<0.001$ and $\left.{ }^{* \star * \star} \mathrm{p}<0.0001\right]$. Red circle indicates the relatedness between $\mathrm{C} 24$ with other treatments, Blue Square shows the relatedness between $\mathrm{C} 48$ and other treatments, Green diamond indicates the relatedness between P24 with other treatments, and empty circle shows the relatedness between P48 with other treatments. The relatedness between other treatments are shown with brackets. 